\title{
Mixed Scenario of the Charged Helium Surface Reconstruction
}

\author{
P. Leiderer • V. Shikin
}

\begin{abstract}
Discussed in the paper is the mixed scenario of charged liquid surface reconstruction when the surface $2 \mathrm{D}$ charge density is close to the saturation. The basic building block of the arising honeycomb structure is shown to be a modifie multielectron dimple.
\end{abstract}

Keywords Liquid helium · Charged surface $\cdot$ Reconstruction

One of the instabilities studied in classical hydrodynamics is the Frenkel-Tonks (FT) instability [1-3] occurring in a threshold manner at the charged liquid surface and resulting in its deformation. A distinctive feature of the FT instability compared with other decay processes: Rayleigh instability of the cylindrical jet [4], Karman trace behind the moving cylinder (sphere) [5], instability of the interface between two liquid media moving relative to each other [6], Taylor vortex instability of viscous liquids between two rotating coaxial cylinders [6], etc. is the possibility of stopping the decay process after which a new metastable state with a finit corrugation amplitude is formed (i.e., a reconstruction occurs). At different times this phenomenon was considered by many authors for dielectric and charged interfaces [7-29]. However, many important details of this process have only been understood quite recently. In the FT instability, only the initial part of the problem (calculation of the dispersion law and its stability analysis) has a universal nature. Further analysis requires different treatments for the dielectric and charged boundaries. In contrast to the dielectric interfaces, the latter case allows for the formation of solitons of a special kind, the

\author{
P. Leiderer ( $\triangle)$ \\ University of Konstanz, 78457, Konstanz, Germany \\ e-mail: Paul.Leiderer@uni-konstanz.de \\ V. Shikin \\ Institute of Solid State Physics of RAS, Chernogolovka, Moscow distr., 142432, Russia
}


multielectron dimples [22, 23]. A less obvious, but actually more important factor resulting in different behaviour of neutral and charged surfaces is the violation of homogeneity of boundary conditions (the surface charge distribution along the interface can become discontinuous). Earlier Mel'nikov and Meshkov showed [24, 26] that the boundary condition inhomogeneity inevitably occurs in the problem of the charged helium surface reconstruction. Therefore, its analysis should account for arising inhomogeneities.

Unfortunately, inhomogeneities predicted in Refs. [24, 26] develop as a periodic system of small "bald" areas of radius $R^{*} \ll a, a=\sqrt{\alpha / \rho g}$, while for $v \ll 1$ the experiment reveals an aperiodic set of individual multielectron dimples with the charged core radius $R \ll a$. In addition, the maximum surface curvature in the central parts of neutral spots has the sign opposite to that observed experimentally [28]. These circumstances hamper evaluation of the feasibility of the model proposed in Refs. $[24,26]$. Later, it was found that in the range of $v \ll 1$ the details of the reconstruction are much more naturally described in terms of individual multielectron dimples [29]. Here and below the boundary population with mobile charges $v$ is define as the ratio

$$
v=n_{s} / n_{s}^{\max }, \quad 2 \pi\left(e n_{s}^{\max }\right)^{2}=\sqrt{\alpha \rho g},
$$

where $\rho$ and $\alpha$ are the density and surface tension, $g$ is the acceleration due to gravity, and $e$ is the electron charge.

In the opposite, most suitable for observations limit $v \leq 1$ where the equipotential language $[16,21]$ and its modificatio $[24,26]$ do not work at all, it was suggested to model the corrugation with inhomogeneous electrostatic potential distribution by a periodic system of multielectron dimples [27]. The analysis of Ref. [27] is based on tight binding approximation. The structure is assumed to consist of roughly estimated free multielectron dimples. By further assuming these quasiparticles to be point-like, the authors model the periodic lattice of charged dimples by employing the results derived for classical Coulomb crystal consisting of point charges [30]. The dimple crystal spacing is approximately equal to the capillary length while its associated energy gain compared with the homogeneous $2 \dot{\mathrm{D}}$ charge distribution is treated in terms of correlations [30]. To comment on the results of Ref. [27], we note that the single dimple problem accurately solved in Ref. [25] yields for the conditions of Ref. [27] the charged spot radius considerably exceeding the lattice spacing given in Ref. [27] (according to Ref. [25], here $R / a \simeq 1.5$ ). The critical conditions for its development is higher than the dynamic stability threshold. Finally, we believe the correlation effects [30] are not directly related to the Coulomb part of the reconstruction problem. Thus, the dimple picture of a periodic reconstruction, being almost the only realistic in the range of $v \leq 1$, contains in the formulation given in Ref. [27] a number of disputable concepts whose presence should be at least understood and which should be modified The details of such an analysis are given in the present report.

1. From our point of view, most questionable are suggestions of authors of Ref. [27] on the Coulomb part of the problem. It is assumed that the development of a periodic corrugation is strongly assisted by the well-known gain in energy occurring when the 2D charged system goes from the gaseous (liquid) state to the crystalline one (Wigner crystal). Such a gain of the correlation origin indeed takes place (e.g. 
see Ref. [30]) as revealed by analysis of the difference between the average Coulomb energy of (i) likely charged point-like particles $\left\langle V_{c}^{l}(r)\right\rangle$ uniformly distributed along the surface with the average density $n_{s}$, and (ii) those arranged in a lattice $\left\langle V_{c}^{s}(r)\right\rangle$ with the same average density

$$
\delta\left\langle V_{c}(r)\right\rangle=\left\langle V_{c}^{l}(r)\right\rangle-\left\langle V_{c}^{s}(r)\right\rangle \simeq+c_{s} e^{2} \sqrt{n_{s}} .
$$

Here the constant $c_{s}$ of the order of unity depends on the lattice type. By employing a formal analogy between the structure of a classical Wigner solid with one electron per cell and the corrugation having spacing $b$ and the charge $q_{0}$ per cell, it is possible to assign to the Coulomb part of the reconstruction problem (as it was done in Ref. [27]) the energy gain of the order of

$$
\delta\left\langle W_{c}(r)\right\rangle \simeq+c_{s} q_{0}^{2} / b>0
$$

However, the hypothesis ( 3 ) can actually be checked and, at least for the employed model of a corrugation involving formation of a set of one-dimensional conducting threads in the practically interesting case $v=1$ is not confirmed Detailed calculations (which can be found in the Appendix) lead to the energy whose sign is opposite to that in (3):

$$
\begin{aligned}
& \delta\left\langle W_{c}(r)\right\rangle=\frac{Q^{2}}{2}(1 / \bar{C}-1 / \tilde{C})<0 \\
& \bar{C}=\frac{S}{4 \pi d}, \quad \tilde{C}=\frac{S}{4 \pi d+4 a \ln (a / \pi R)}
\end{aligned}
$$

where $S$ is the capacitor area, $R$ is the single thread radius, $2 a$ is the corrugation period. In addition to the sign which is opposite to that of (3), the formula (4) has one more qualitatively important property: here the energy gain depends explicitly on the length $R$ measuring the charges localization length in a separate groove. This length is determined self-consistently by requiring the minimum of the corrugated system total energy. In the approximation used in Ref. [27], the Coulomb part of the problem which does not contain $R$ slips out of the self-consistency condition which should not be allowed. In the rest of the paper we follow the definitio of $\delta\left\langle W_{c}(r)\right\rangle$ given by (4).

2. Here are some remarks before the study of the corrugation problem for $v \leq 1$. First of all, the total charge $Q$ at the liquid-vapor interface with area $S \sim L^{2}$ is constant:

$$
Q=\text { const }=e n_{s}^{\max } L^{2}
$$

Perhaps a trivial point, but the problem formulation should for definitenes contain some comments on the initial stage of the decay. It develops within the dynamic equipotential scenario [16], until under the conditions of (6) the growth of the corrugation amplitude results in the break of continuity in the charge distribution. In the limit of small $v$ the inevitable appearance of continuity breaks was noted by authors of Refs. [24, 26]. However, their reasoning is essentially universal in nature and is applicable for arbitrary values of $v$. If one assumes that the spatial period of developing 
instability is not changed with time (in the general theory of spinodal kinetics relevant to the problem considered here this assumption is not true [31]), the net charge splits into a system of $N$ individual clusters of charge $Q_{0}$ per unit length

$$
N=L / 2 a, \quad Q_{0} \simeq 2 a e n_{s}^{\max }
$$

each of which is the nucleus of a separate one-dimensional (for simplicity) dimple residing at its center. It should be noted that the unstable state could decay in a purely fluctuationa (binodal) way just as in the case of $v \ll 1$ (see Ref. [29]). However, in the general case of arbitrary $v$ the dimple charge remains undefine in this scenario. The decay having a spinodal initial and binodal fina stages (we call it "intermediate") does not contain this uncertainty.

In the problem of findin the corrugated surface state in the critical electric fiel $E_{+}^{\max }$ with the charge $Q_{0}$ (7) per cell, one should verify the ability of the deformed helium surface to hold the critical charge (perhaps taking into account the non-linear phenomena) and check the validity of inequality

$$
\bar{W}\left(E_{+}^{\max }, Q\right) \geq \tilde{W}\left(E_{+}^{\max }, Q_{0}, b\right), \quad E_{+}^{\max }=4 \pi e n_{s}^{\max }
$$

where $n_{s}^{\max }$ is taken from (1), $\bar{W}\left(E_{+}^{\max }, Q\right), \tilde{W}\left(E_{+}^{\max }, Q_{0}, b\right)$ are the total energies of the uniform and corrugated helium surface states, $b$ is the lattice spacing which is generally different from the capillary length $a$. There are no reasons to require coincidence of energies in (8) since we do not consider the actual binodal transition from a fla to corrugated state. Nevertheless, it is desirable to have the energy $\tilde{W}\left(E_{+}^{\max }, Q_{0}, b\right)$ to be less than $\bar{W}\left(E_{+}^{\max }, Q\right)$, since otherwise the general picture of the system evolution could not be reasonably interpreted.

Bearing in mind the above arguments and results derived in the Appendix, the problem of one-dimensional corrugation is reduced to calculating the energy difference $\bar{W}\left(E_{+}^{\max }, Q\right)-\tilde{W}\left(E_{+}^{\max }, Q_{0}, b\right)(8)$. Note that the one-dimensional scenario is not only a convenient model but also an experimentally observed state of the corrugated surface [28]. In addition, this is the least stable type of corrugation. Laplace pressure stabilizing the picture is twice as low in the one-dimensional case as in two dimensions. The existence of a solution yields an estimate from below for the corrugation stability.

The initial energy functional (per unit thread length) for a one-dimensional corrugation with the amplitude $\xi(x)$

$$
\begin{aligned}
& \delta \tilde{W}\left(E_{+}^{\max }, R, a\right)=\int_{-a}^{+a} d x\left[\frac{\alpha}{2}\left[(\nabla \xi)^{2}+\kappa^{2} \xi^{2}\right]+e E_{+}^{\max } \delta n(x) \xi(x)\right]+\delta\left\langle W_{c}(r)\right\rangle \\
& \delta\left\langle W_{c}(r)\right\rangle=2 e^{2} n_{s}^{2} a^{2} \ln \frac{a}{\pi R} \\
& \xi(x)=\zeta(x)-\langle\zeta\rangle, \quad \delta n(x)=n(x)-n_{s}, \quad \kappa=\sqrt{\rho g / \alpha}
\end{aligned}
$$

represents the system energy counted from the energy of uniformly charged fla liquid surface and written for one lattice cell. Its Coulomb part is taken in the form of 


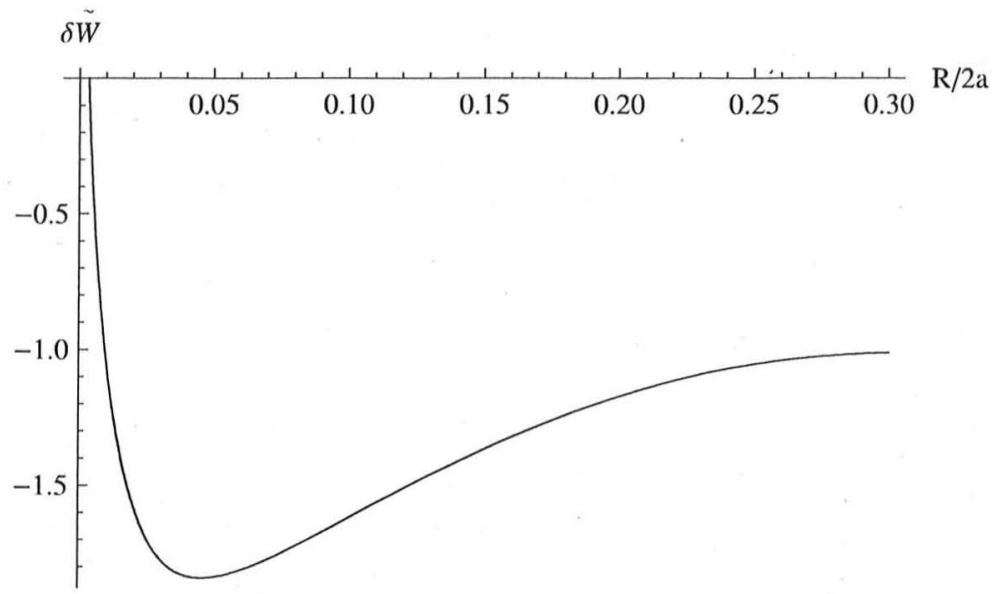

Fig. 1 Energy gain $\delta \tilde{W}(9)$ in units of $e^{2} n_{s}^{2} a^{2}$ as a function of the electron spot radius. The negative minimum corresponds to the optimal value of $R / a$. The plot has been calculated with the following values of physical parameters: $\alpha=0.36 \mathrm{erg} / \mathrm{cm}^{2}, \rho=0.146 \mathrm{~g} / \mathrm{cm}^{3}, g=980 \mathrm{~cm} / \mathrm{s}^{2}, E_{+}^{\max }$ is define in (8)

(4) allowing variational solution for the problem of findin the length $R$ if the charge density $n(x)$ in a periodic system of dimples is split into individual stripes each having some density distribution $n_{0}(x)$ (for example, a Gaussian one)

$$
n(x)=\sum_{l} n_{0}(x-2 l a), \quad n_{0}(x)=n_{0} \exp \left(-x^{2} / R^{2}\right), \quad \sqrt{\pi} n_{0}=\frac{n_{s} a}{\operatorname{erf}(a / R) R}
$$

$(\operatorname{erf}(x)$ is the known special function) normalized according to (7). Average values of the variables $\zeta(x), n(x)$ are subtracted from their initial definition according to the general structure of the functional (9).

The functional (9) has the standard form for the theory of multielectron dimples. The deformation-related part written in square brackets makes the reduction of the parameter $R / a$ as well as the growth of liquid surface deformation due to local electron pressure energetically favorable. The Coulomb component $\delta\left\langle W_{c}(r)\right\rangle$ hinders this process. The interplay between these factors results in the appearance of a negative minimum in the $\delta \tilde{W}\left(E_{+}^{\max }, R / a\right)(9)$ dependence which determines the equilibrium value of the parameter $R / a$. To get the numerical values, one should solve the liquid surface mechanical equilibrium equation for $\xi(x)$ assuming $n(x)(10)$ to be a known function, then calculate the integrals (9) with these distribution, and finall obtain the energy (9) dependence on the variational parameter $R / a$. For the external parameters corresponding to $v=1$ this picture is presented in Fig. 1 .

The functional minimum is reached at $R / a=0.1$ and is negative. In other words, the corrugated state is more favorable than the fla one. The corrugation is stabilized in the range of $R / a \ll 1$, although the obtained numbers should only be considered as a rough estimate because the values of $\nabla \xi(x) \leq 1$ are not parametrically small, as follows from the data on the one-dimensional dimple profil presented in Fig. 2. Also shown for comparison in the same figur is the individual free dimple profile Obviously, the linear theory corresponding to the bilinear energy functional (9) requires 


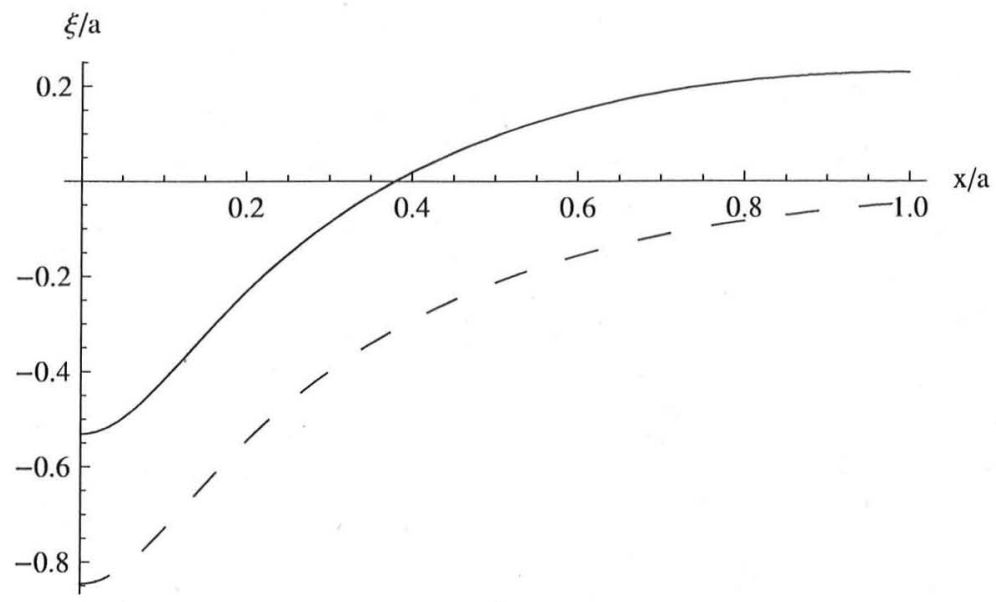

Fig. 2 One-dimensional dimple profile for a single free dimple (dashes) and a periodic set of dimples (solid line)

quantitative corrections (it follows from the figur that the inequality $\nabla \xi(x)<1$ used to derive (9) is only poorly met). However, qualitatively the mixed reconstruction scenario is self-consistent (starting from the assumption on the presence of continuity breaks in the equilibrium distribution $n(x)$ we finall obtain the picture confirmin the initial assumptions of the theory).

A similar problem for the honeycomb helium surface corrugation yields

$$
\begin{aligned}
& E_{\perp} \xi^{\prime \prime}(0)=\frac{3 \pi Q}{4 R^{3}}, \quad R=\pi^{2} \alpha / E_{\perp}^{2}, \quad \xi(0)=\sum_{n=1}^{\infty} \tilde{\xi}_{n}, \\
& \tilde{\xi}_{n}=-\frac{A f\left(\mu_{n}, R / a\right)}{1+\mu_{n}^{2} J_{0}^{2}\left(\mu_{n}\right)}, \quad f(x)=\frac{\sin x-x \cos x}{x^{3}}, \quad A=\frac{3 Q E_{\perp}}{\pi \alpha}
\end{aligned}
$$

where $\mu_{n}$ are the roots of equation $\partial J_{0}\left(\mu_{n} r / a\right) /\left.\partial r\right|_{r=a}=0$.

By assuming in the general formulae (11) $E_{\perp}=E_{+}=4 \pi e n_{s}^{\max }$, one can estimate the most interesting dimple parameters:

$$
\frac{R_{\text {crit }}}{a} \simeq 0.4<1, \quad \frac{\xi_{\text {crit }}(0)}{a} \simeq 0.3<1
$$

Hence, just as in the comments to Figs. 1 and 2, the mixed scenario of surface reconstruction seems to be quite acceptable here.

To sum up, we conclude that the mixed scenario of the charged liquid surface reconstruction under the condition of $v=1$ involving the formation of a periodic system of charged dimples has a self-consistent nature. The period of arising structure is determined by the dynamical instability of charged liquid surface. Development of this instability divides the surface charges into a periodic system of clusters localized in every cell of a one-dimensional or a honey-comb corrugation. When approaching the metastable state, the charged cell cores become rather small $(R / a \ll 1)$ while 


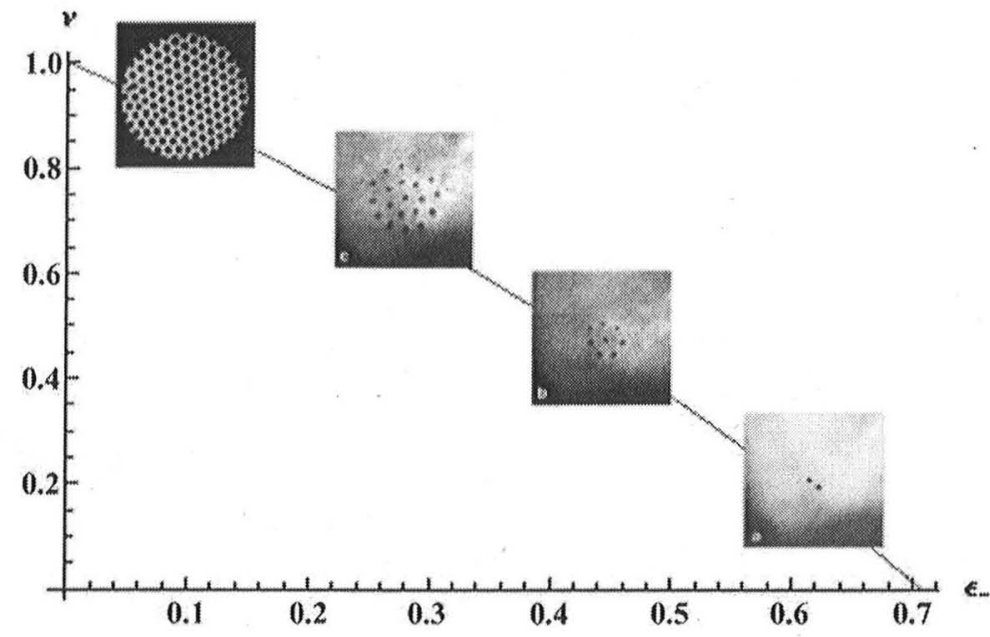

Fig. 3 Phase diagram in the $\left(\nu, \epsilon_{-}\right)$coordinates: upper left part-a configuratio close to honeycomb crystal covering the entire charged surface of the liquid; lower right corner-individual multielectron dimples; middle part—clusters of individual dimples; the pictures are taken from Refs. [27, 28]

the corrugation amplitude is of the order of the capillary length (stiff reconstruction regime according to the classificatio of Ref. [12]). The mixed scenario reveals no hysteresis phenomena typical of nonlinear equipotential theories (which is not surprising because of the employed linear approximation (9) for energy).

Bearing in mind the above arguments as well as the earlier results obtained in Refs. $[28,29]$ one can suggest the qualitative phase diagram for the fla to corrugated charged helium surface transition in the $\left(v, \epsilon_{-}\right)$coordinates shown in Fig. 3. Here $v$ is the fillin factor from (1), and $\epsilon_{-}$is the positive dimensionless electric fiel above the charged helium surface (zero corresponding to complete saturation).

Acknowledgement This work was partly supported by the RFBR grant 09-02-00894a.

\section{Appendix}

The statements from Ref. [30] concerning the properties of the difference

$$
\delta\left\langle V_{c}(r)\right\rangle=\left\langle V_{c}^{l}(r)\right\rangle-\left\langle V_{c}^{s}(r)\right\rangle
$$

are based on the analysis of the expression

$$
\begin{aligned}
\delta\left\langle V_{c}(r)\right\rangle & =e^{2} \lim _{\mathbf{x} \rightarrow 0} \sum_{l}\left[\frac{1}{|\mathbf{x}-\mathbf{x}(l)|}-\frac{1}{|\mathbf{x}|}\right] \\
\mathbf{x}(l) & =l_{1} \mathbf{a}_{1}+l_{2} \mathbf{a}_{2},
\end{aligned}
$$

where $\mathbf{a}_{1}$ and $\mathbf{a}_{2}$ are the primitive translation vectors in the considered lattice. 
The sums in (14) diverge already because the classical proper energy of every electron in the definitio (14) is infinite However, the Fourier analysis and the important condition of the system neutrality as a whole (which is ensured by introducing the screening electrode) allow to reduce the difference of the sums to a finit expression of the form (e.g., see Ref. [30])

$$
\delta\left\langle V_{c}(r)\right\rangle=N c_{s} e^{2} \sqrt{a_{c}} / 2,
$$

where $N$ is the total number of electrons in the 2D system, $a_{c} \simeq n_{s}^{-1}$ is the unit cell area, $c_{S}$ is a constant of the order of unity depending on the lattice type.

An elegant and rather general result (16) gives an idea on the nature of Coulomb crystallization of electrons above helium yielding the crystal ground state energy in this problem compared to the gaseous (liquid) phase.

Let us now turn to the known electrostatic problem [32] on the fiel and energy of a set of charged threads above a conducting screen, i.e. the problem of a fla capacitor with a solid or mesh-like electrodes. Here the electrostatic potential has the form

$$
\varphi(z)=-2 a \sigma \ln \left(\frac{\sin [\pi(z-i d) / a]}{\sin [\pi(z+i d) / a]}\right), \quad z=x+i y
$$

where $a$ is the lattice spacing, $\sigma$ is the mesh charge per unit area, $d$ is the distance between the mesh and the screen. Just as in (14), the divergencies at infinit cancel each other. The fiel is concentrated within the capacitor, the mesh fiel becoming uniform at distances of the order of its period $2 a \ll 2 d$ where $2 d$ is the distance between the plates. The total electrostatic energy (the counterpart of (14)) calculated in the field-theoretica approach (squared electric fiel integrated over the capacitor volume) proves to be divergent due to the potential $\varphi(z)(17)$ singularity at the threads. This property requiring cutoff at the thread radius $R \ll a$ does not appear in the fina result (16).

Capacities $\bar{C}, \tilde{C}$ per unit system area after using (17) can be calculated to be

$$
\bar{C}=\frac{1}{4 \pi d}, \quad \tilde{C}=\frac{1}{4 \pi d+2 a \ln (a / 2 \pi R)} .
$$

Here $R \ll a / 2 \pi$ is the radius of a single thread.

The counterpart of expression (14) is obvious:

$$
\delta\left\langle W_{c}(r)\right\rangle=\frac{Q^{2}}{2}(1 / \bar{C}-1 / \tilde{C})
$$

This difference has the sign opposite to that of (16) and depends on particular characteristics of a single thread.

In the arising alternative ((18) vs. (15)) we prefer the expression (18) based on the following qualitative arguments. The procedure of findin the difference (13)-(16) assumes that each charge both in liquid and solid states has the same structure (e.g., point-like). As a result, all single-particle singularities in electrostatic energy related to the point-like structure of original charges are the same in both phases and cancel out when the subtraction (13), (14) is realized. 
In the reconstruction problem we are now interested in comparing the energies of two capacitors with different locally continuous charge distributions, i.e. a homogeneous one and a periodic distribution of charged threads possessing the same 2D electron density. Obviously, the electrostatic energy of these threads increases with the reduction of the thread radius, just as suggested by (18).

As to the correlation energy (14)-(15), it can also arise, in principle, in the scenario (17)-(18) if the surface reconstruction is accompanied with the liquid-Coulomb crystal phase transition. However, under the conditions we are interested in both homogeneous and corrugated electronic configuration at the microscopic level are liquid. Therefore, accounting for phase-related correlations in the algorithm (17)-(18) is not required.

\section{References}

1. Ya. Frenkel, Zs. der Sowietunion 8, 675 (1935)

2. Ya. Frenkel, ZhETF 6, 347 (1936)

3. T. Tonks, Phys. Rev. 48, 562 (1935)

4. Strett, Theory of Sound, vol. II (Gosizdat, Moscow, 1955)

5. G. Batchelor, Introduction to Hydrodynamics (Mir, Moscow, 1973)

6. L. Landau, E. Lifshitz, Hydrodynamics (Nauka, Moscow, 1986)

7. J. Melcher, Field-Coupled Surface Waves (MIT Press, Cambridge, 1963)

8. G. Taylor, A. McEwan, J. Fluid Mech. 22, 1 (1965)

9. M. Cowley, R. Rosenswieg, J. Fluid Mech. 30, 671 (1967)

10. V. Zaitsev, M. Shliomis, Dokl. Acad. Nauk 188, 1261 (1969)

11. L. Gor'kov, D. Chernikova, Pis'ma v ZhETF 18, 119 (1973)

12. M. Shliomis, Usp. Fiz. Nauk 112, 437 (1974)

13. D. Chernikova, ZhETF 68, 250 (1975)

14. E. Kuznetsov, M. Spektor, ZhETF 71, 262 (1976)

15. A. Volodin, M. Khaikin, V. Edelman, Pis'ma v ZhETF 23, 524 (1976)

16. L. Gor'kov, D. Chernikova, Dokl. Acad. Nauk USSR 228, 829 (1976)

17. A. Volodin, M. Khaikin, V. Edelman, Pis'ma v ZhETF 26, 707 (1977)

18. P. Leiderer, Phys. Rev. B 20, 4511 (1979)

19. P. Leiderer, M. Wanner, Phys. Lett. A 73, 189 (1979)

20. M. Wanner, P. Leiderer, Phys. Rev. Lett. 42, 315 (1979)

21. H. Ikezi, Phys. Rev. Lett. 42, 1688 (1979)

22. V. Shikin, P. Leiderer, Pis'ma v ZhETF 32, 439 (1980)

23. V. Shikin, P. Leiderer, ZhETF 81, 134 (1981)

24. V. Melnikov, S. Meshkov, Pis'ma v ZhETF 33, 222 (1981)

25. V. Melnikov, S. Meshkov, ZhETF 81, 951 (1981)

26. V. Melnikov, S. Meshkov, ZhETF 82, 1910 (1982)

27. H. Ikezi, R. Gianetta, P. Platzman, Phys. Rev. B 25, 4488 (1982)

28. P. Leiderer, W. Ebner, V. Shikin, Surf. Sci. 113, 405 (1982)

29. V. Shikin, Pis'ma ZhETF 78, 930 (2003)

30. L. Bonsall, A. Maradudin, Phys. Rev. B 15, 1959 (1977)

31. A. Olemskii, I. Koplyk, Usp. Fiz. Nauk 165, 1105 (1995)

32. F. Morse, G. Feshbach, Methods of Theoretical Physics, vol. 2 (Izdat. Inostr. Lit., Moscow, 1960), $896 \mathrm{pp}$ 